Boxplot to show distribution of CMR T2 mapping values representing myocardial oedema at different stages of myocarditis compared with healthy volunteers

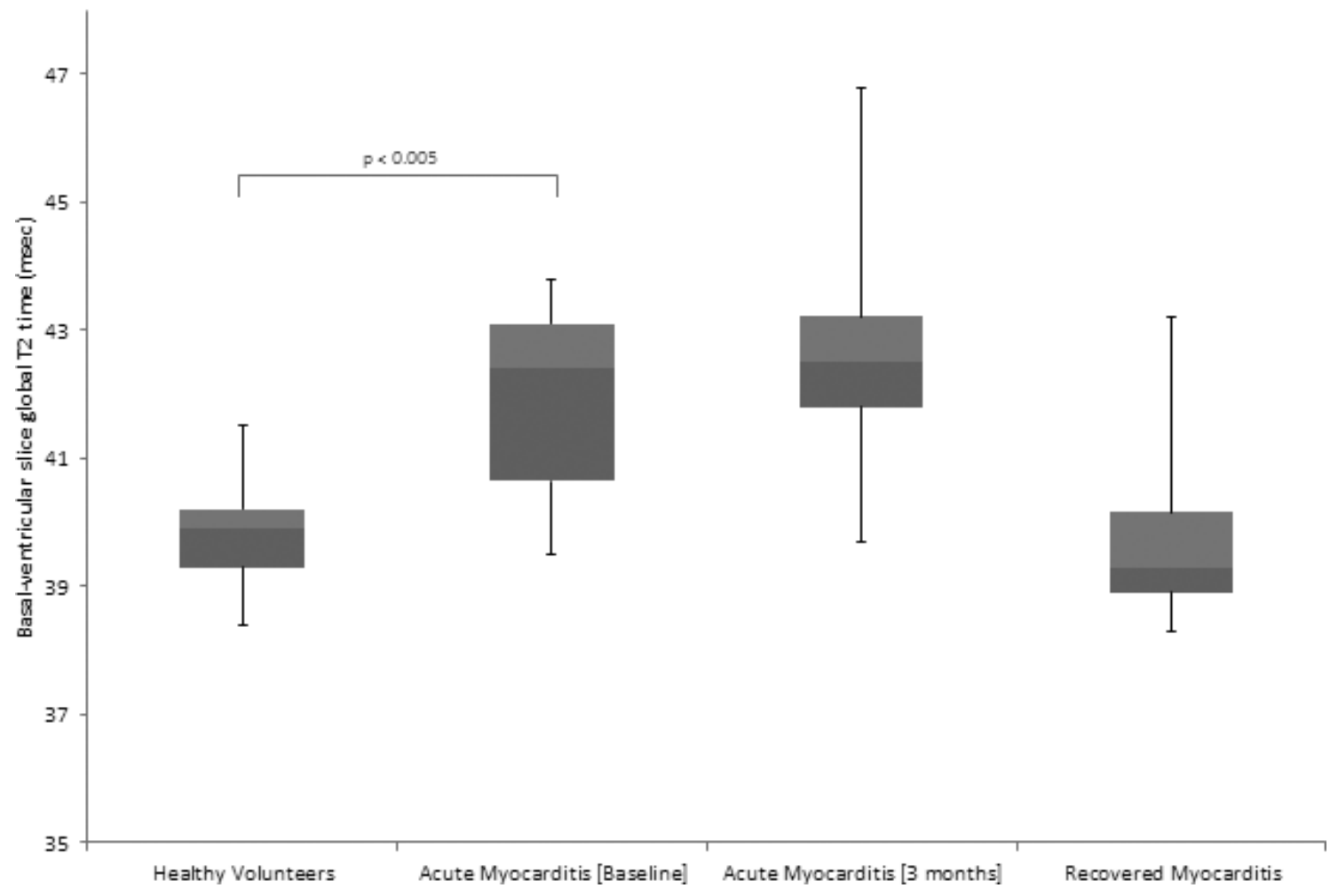

Abstract 027 Figure 2 Boxplot showing distribution of CMR T2 mapping values at different stages of acute myocarditis compared with healthy volunteers. The centreline in each box represents the median, whereas the lower and upper limits of each box represent the 25th and 75th percentiles, respectively. Whiskers extend to the most extreme observations.

\section{ROUTINE IDENTIFICATION OF HYPOPERFUSION IN CARDIAC AMYLOIDOSIS BY MYOCARDIAL BLOOD FLOW MAPPING}

${ }^{1}$ Ana Martinez-Naharro, , ${ }^{1}$ Daniel S Knight, ${ }^{1}$ Tushar Kotecha, ${ }^{1}$ Rohin Francis, ${ }^{1}$ Giulia Zumbo, ${ }^{2}$ Thomas A Treibel, ${ }^{3}$ Jannike Nickander, ${ }^{3}$ Raquel Themudo, ${ }^{1} J u l i a n$ D Gillmore, ${ }^{3}$ Martin Ugander, ${ }^{2} J a m e s$ C Moon, ${ }^{4}$ Hui Xue, ${ }^{4}$ Peter Kellman, ${ }^{1}$ Philip N Hawkins, 'Marianna Fontana. 'National Amyloidosis Centre, University College London, Royal Free Hospital, London, UK; ${ }^{2}$ Karolinska Insitutet, Sweden; ${ }^{3}$ Barts Heart Centre, West Smithfield, London, UK; ${ }^{4}$ National Heart, Lung and Blood Institute, National Institutes of Health, Bethesda, Maryland, USA

\subsection{6/heartjnl-2017-311399.28}

Background Cardiac involvement is the main driver of outcome in systemic amyloidosis, but the relationship between amyloid deposits and cellular injury is not well understood. The simple explanation of physical, mechanical replacement of parenchymal tissue seems insufficient, and preliminary studies support the hypothesis that myocardial hypoperfusion could contribute to cell damage in amyloidosis. The aim of this study was: 1) To assess feasibility of fully automated pixelwise rest myocardial blood flow (MBF) mapping in cardiac amyloidosis during routine clinical scans; 2) To assess the prevalence of myocardial hypoperfusion and correlation with amyloid deposits and disease severity.

Methods Patients $(\mathrm{n}=56)$ with systemic amyloidosis and healthy volunteers $(n=16)$ were recruited. All subjects underwent CMR at 1.5T (Siemens) with standard SSFP cine imaging, Phase Sensitive Inversion Recovery Reconstruction Late Gadolinium Enhancement (PSIR-LGE), T1 mapping, Extracellular Volume (ECV) mapping and rest MBF mapping. Results The pixel-wise MBF maps for all slices were generated automatically in all patients within $2.5 \mathrm{~min}$ after image acquisition. Myocardial perfusion was globally reduced in patients with cardiac amyloidosis compared to healthy volunteers $(0.66$ $\pm 0.26 \mathrm{ml} / \mathrm{min} / \mathrm{g}$ vs $0.84 \pm 0.19 \mathrm{ml} / \mathrm{min} / \mathrm{g}, \mathrm{p}<0.05)$. Myocardial perfusion inversely correlated with amyloid burden measured as extracellular volume fraction $(\mathrm{r}=-0.46, \mathrm{p}<0.001)$ (figure 1) and with the transmurality of LGE (no LGE $0.88 \pm 0.18 \mathrm{ml} /$ $\mathrm{min} / \mathrm{g}$, subendocardial LGE $0.73 \pm 0.28 \mathrm{ml} / \mathrm{min} / \mathrm{g}$ and transmural LGE $0.58 \pm 0.20 \mathrm{ml} / \mathrm{min} / \mathrm{g}, \mathrm{p}<0.01$ ) (figure 2). There was a correlation between myocardial perfusion and markers of systolic dysfunction (EF, $\mathrm{r}=0.39, \mathrm{p}<0.01)$ as well as blood biomarkers (NT-proBNP, $\mathrm{r}=-0.41, \mathrm{p}<0.01$ and Troponin $\mathrm{T}$, $\mathrm{r}=-0.41, \mathrm{p}<0.01$ ), current reference prognostic markers in cardiac amyloidosis. There was no significant correlation between myocardial perfusion and native T1 values $(r=-0.07$, $\mathrm{p}=0.59$ ).

Conclusions Myocardial perfusion can be measured in cardiac amyloidosis during routine clinical scans with fully automated MBF mapping. Myocardial hypoperfusion at rest is highly prevalent in subjects with cardiac amyloidosis, and correlates with the degree of amyloid infiltration and disease severity. 